\title{
Les visées discursives dans les discours du MNLA au Mali
}

Nanourougo Coulibaly

\section{(2) OpenEdition}

1 Journals

Édition électronique

URL : http://journals.openedition.org/rhetorique/579

DOI : $10.4000 /$ rhetorique. 579

ISSN : 2270-6909

Éditeur

UGA Éditions/Université Grenoble Alpes

Édition imprimée

ISBN : 978-2-37747-029-7

Référence électronique

Nanourougo Coulibaly, «Les visées discursives dans les discours du MNLA au Mali », Exercices de rhétorique [En ligne], 10 | 2017, mis en ligne le 21 décembre 2017, consulté le 04 octobre 2020. URL : http://journals.openedition.org/rhetorique/579 ; DOI : https://doi.org/10.4000/rhetorique.579

Ce document a été généré automatiquement le 4 octobre 2020.

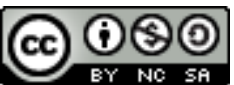

Les contenus de la revue Exercices de rhétorique sont mis à disposition selon les termes de la Licence Creative Commons Attribution - Pas d'Utilisation Commerciale - Partage dans les Mêmes Conditions 4.0 International. 


\title{
Les visées discursives dans les discours du MNLA au Mali
}

\author{
Nanourougo Coulibaly
}

Au début de l'année 2012, le paysage politique malien a été marqué par la résurgence de l'irrédentisme touareg qui a réclamé par le moyen des armes l'indépendance du nordMali. Cette action est conduite par le Mouvement National pour la Libération de l'Azawad (MNLA). Créé en octobre 2011, le MNLA a déclenché les hostilités par l'attaque des positions de l'armée malienne et la prise d'Aguelhok dans le nord du pays. Outre le crépitement des armes, cette résurgence de la guerre sécessionniste menée par une partie des ressortissants du nord se mène par le moyen de communiqués de presse, de déclarations, de conférences de presse et autres adresses à l'occasion de commémoration d'évènements nationaux ou non. C'est cet ensemble de productions discursives que la présente réflexion se propose d'analyser à partir des régularités formelles et énonciatives qui la caractérisent et qui en font le carrefour d'une diversité de registres discursifs mobilisés pour atteindre certains résultats. L'étude part de l'hypothèse que le discours du mouvement rebelle est traversé par une diversité de modalités du dire faisant intervenir une multiplicité de registres pour répondre aux visées discursives du locuteur. Il s'agira de montrer comment le discours produit par le MNLA, à partir des différents appareils formels qui y interagissent, entremêle les registres polémique, élégiaque (la lamentation), délibératif pour justifier l'entrée en guerre, se positionner dans l'espace public et s'assurer des soutiens. Pour traiter cette problématique, l'analyse est constituée de trois temps forts: le premier précisera les notions théoriques et méthodologiques, tandis que le second et le troisième reviendront sur l'exploration du corpus, constitué d'une dizaine de discours des communicants du MNLA. 


\section{Discours, registres discursifs, éléments de contexte du corpus}

2 Les communiqués de presse et autres déclarations des communicants du MNLA présentent certaines régularités lexicales, discursives et énonciatives qui orientent l'observateur sur les stratégies de positionnement du mouvement rebelle sur l'espace public. Ces choix discursifs nous amènent à nous référer à la théorie des appareils formels formulée par Émile Benveniste dans le cadre de ses réflexions sur l'énonciation et reprise par Gilles Philippe pour qui «le propre d'un appareil formel serait d'être réductible à un ensemble de faits langagiers co-orientés, ponctuels mais aptes à modifier le statut de la totalité du discours où ils apparaissent ${ }^{1} »$. Il poursuit en affirmant que «l'appareil formel est défini à la fois par la présence prévisible ou par l'absence prévisible de certains traits langagiers » et que "on appellera "pivot" de l'appareil formel le ou les fait(s) de langue dont l'apparition est non seulement prévisible mais obligatoire ». Le discours ainsi produit est alimenté par une diversité d'appareils formels déroulant chacun un registre spécifique si on s'accorde qu'un registre est un appareil formel exigé par l'expression de telle ou telle position affective du sujet par rapport à l'objet dénoté ${ }^{2}$. Sans entrer ici dans la discussion critique sur la notion de registre discursif, on se contentera de noter l'existence d'une diversité d'approches, et de recommander le numéro 124 de la revue Langage et société ${ }^{3}$, ainsi que les travaux de Pierre Achard relatif à la sociologie du langage : notre lecteur aura ainsi un large aperçu sur la question des registres qui interviennent aussi bien en sociolinguistique, en analyse textuelle qu'en analyse de discours, chaque champ du savoir esquissant ses définitions et ses spécificités.

3 Pour notre part, nous emploierons la notion de registre dans le cadre de l'analyse du discours. Pour citer Dominique Maingueneau :

le terme «registre » peut a priori désigner n'importe quel ensemble de traits linguistiques régulièrement associés dans le discours et qui ne se laissent pas enfermer dans les limites d'un genre4.

4 C'est dire que le trait spécifique du registre est sa transversalité ou sa transgénéricité. C'est d'ailleurs cette spécificité qui fonde l'approche développée par Maingueneau. Ce dernier emploie le terme de registre pour qualifier des « unités transverses », c'est-àdire qu'on ne peut circonscrire dans un genre ou un type de discours. De manière très générale il propose trois sortes de registres déterminés «en fonction des critères qui président à leur définition: critères linguistiques, fonctionnels ou communicationnels ». Ces trois critères correspondent à l'approche pragmatique développée par Marc Bonhomme :

Le principe définitoire des registres est plutôt pragmatique, ceux-ci reposant sur des macro-actes qui régulent les productions verbales, tout en orientant leur interprétation. Ainsi, chaque registre est centré sur une tonalité illocutoire spécifique : faire rire de $x$ pour le comique, se moquer de $x$ pour le satirique, faire savoir $x$ pour le didactique, conseiller $x$ pour le délibératif, se plaindre de $x$ pour l'élégiaque, être ému par $x$ pour le pathétique, etc. ${ }^{5}$.

5 Lier le registre à la tonalité illocutoire revient à admettre qu'un acte de discours est déterminé par le contexte lequel, à son tour, impose un certain usage des ressources linguistiques et donc une manipulation orientée du matériau verbal en relation avec le projet de communication qui sous-tend l'acte discursif. 
6 Sur ces bases, quelle est la spécificité du contexte d'émergence du discours du MNLA et quelles en sont les implications discursives? Cette parole émerge dans un contexte de remise en cause de l'ordre établi. Les locuteurs recourent au langage dans un processus de contestation, de protestation sur fond de rhétorique de polarisation où le groupe est en lutte ouverte avec un adversaire. Au centre de ce combat se trouve un enjeu capital. Le mouvement revendique un "peuple de l'Azawad» opposé à l'Etat malien. L'enjeu étant l'indépendance de "l'Azawad». Les productions discursives du MNLA, mouvement rebelle constitué par la fusion ${ }^{6}$ de plusieurs organisations irrédentistes (Mouvement National de l'Azawad ou MNA, et le Mouvement Touareg du Nord Mali ou MTNM) sont marquées par l'antagonisme des intérêts et des positions tenues et surtout exprimées par ce mouvement avec les positions et les intérêts de l'autorité centrale de Bamako ${ }^{7}$. Cet antagonisme porte fondamentalement sur la volonté du mouvement de créer un État indépendant ayant pour territoire la partie septentrionale du Mali dénommé "Azawad» afin de, selon un communiqué signé du porte-parole du mouvement, "sortir le peuple de l'Azawad de l'occupation illégale du territoire azawadien par le Mali ${ }^{8} »$. Il faut savoir aussi que l'irrédentisme touareg ${ }^{9}$ date depuis la période d'avant les indépendances ${ }^{10}$.

7 Depuis les indépendances, la République du Mali est confrontée à un problème de rébellion ${ }^{11}$ menée par les populations touarègues que tous les régimes successifs ont tenté de résoudre. Les discours considérés ici s'inscrivent dans la stratégie de communication du mouvement rebelle. Le constat est que la production langagière se déploie dans une relation entre trois termes : l'émetteur, l'interlocuteur / l'adversaire et le public visé. De ces interrelations se dégagent trois instances ou identités discursives qui sont le locuteur et sa communauté, présentés comme victime, l'adversaire qui discursivement apparaît comme le mal parfait et enfin le témoin dont l'arbitrage est régulièrement sollicité. Ce tiers est représenté dans le corpus par la communauté internationale. La parole évolue alors entre interpellation, plainte, accusation et dénonciation, ainsi que construction d'une communauté victime dont les qualités sont parfois mises en évidence avec un accent laudatif. Par cette dynamique, le discours du MNLA met en jeu une diversité de modalités ou de traits formels que l'on pourrait résumer comme étant une mise en œuvre d'un arsenal discursif à visées multiples. D'un côté, il vise à rejeter l'adversaire et à susciter colère et indignation contre lui. Mais d'un autre côté, l'enjeu est aussi de créer l'empathie autour de soi en jouant la victime ou en se montrant irréprochable. Chacune de ces orientations discursives se manifeste dans des régularités formelles qui diffèrent et qui relèvent de plusieurs registres discursifs que les locuteurs mobilisent. On peut alors dire que la spécificité du cadre de production des discours du MNLA engendre le recours systématique à des formes grammaticales données, un type d'unités lexicales et des modalités énonciatives spécifiques.

\section{Les registres en jeu dans le discours du MNLA}

Les marques linguistiques dans les productions discursives qui forment le corpus de cette étude leur confèrent des traits stylistiques à partir desquels on peut déduire le registre discursif dont il relève. 


\section{Le registre polémique}

9 S'il y a un registre immédiatement perceptible dans le discours du MNLA, s'est bien le registre polémique. Il s'illustre par certains traits linguistiques spécifiques. La définition du discours polémique par Catherine Kerbrat-Orecchioni est : « un discours disqualifiant, c'est-à-dire qu'il attaque une cible (...) et qu'il met au service de cette visée pragmatique dominante - discréditer l'adversaire, et le discours qu'il est censé tenir - tout l'arsenal de ses procédés rhétoriques et argumentatifs ${ }^{12} »$. Une meilleure compréhension du registre polémique est donnée par M. Bonhomme qui en décrit le script $^{13}$. Cette structure du registre polémique est présente dans une frange importante $\mathrm{du}$ discours du MNLA et les parties en présence sont le mouvement rebelle (en tant que polémiste), la cible (le gouvernement malien) et le tiers (comprenant le peuple de l'Azawad et la communauté internationale). Le polémiste procède à une attaque méthodique de la cible en l'accusant de maux qui ont une certaine résonance dans l'opinion internationale :

$\mathrm{Au}$ début des hostilités militaires, le gouvernement malien avait déjà planifié sa stratégie génocidaire contre les populations civiles de l'Azawad, et l'acte de ce mercredi 22 février 2012, n'est que la traduction de son arrogance en prouvant ainsi qu'il est un État terroriste totalitariste. Le terrorisme, tient de la règle de la terreur et de la torture des personnes sans défense. Faute de mobiliser et motiver ses fantassins sur les champs de combat contre l'armée révolution [sic] du MLNA ${ }^{14}$.

Ce communiqué porte les marques de l'accusation formulée par les communicants du MNLA à l'encontre du "gouvernement malien» qui est présenté comme un «État terroriste totalitariste», ayant planifié "sa stratégie génocidaire» "contre les populations de l'Azawad». Par ailleurs, selon le Communiqué du 8 mars 2012 lors de la célébration de la journée internationale de la Femme, le mouvement rebelle déclare que «l'État Malien à travers ses services de sécurité s'adonne à la violation des droits humains, à travers la torture, les assassinats, et l'arrestation arbitraire des populations civiles, sans défense ${ }^{15} »$.

11 L'observation de cette tendance discursive permet de dégager un propos à deux entrées dont l'une vise le sujet attaqué et l'autre est liée à un public dont le locuteur veut obtenir l'adhésion. Cette tendance se confirme dans le texte de la "Déclaration du Conseil Révolutionnaire » faite à Kidal, jeudi 19 septembre 2013.

12 Ces différentes adresses publiques visent le gouvernement malien protagoniste $d u$ conflit malien et l'accusation a pour fondement doxique la thématique du terrorisme, des droits de l'homme, la protection des minorités et celle de la femme longtemps marginalisée et stigmatisée. Fondement doxique, car le locuteur construit son attaque en mobilisant la doxa de l'époque. Ce trait du registre polémique a été bien mis en valeur par Ruth Amossy :

Le discours polémique utilise des topiques qui éloignent l'auditoire de la thèse ou de la personne de l'autre; il manipule les affects, mais aussi les axiologiques qui suscitent un sentiment moral négatif à l'égard de l'opposant ${ }^{16}$.

13 Ainsi, lorsqu'il convoque le "droit de manifester » en dénonçant la «féroce répression des manifestantes de Kidal qui n'ont fait qu'user d'un droit garanti par les lois internationales, notamment "celui de manifester" ", il attaque non seulement l'adversaire désigné mais il essaie surtout de mobiliser les défenseurs des droits humains à partir de l'imaginaire qui fonde leurs actions. Par ailleurs, le rappel du genre des victimes de la répression n'est pas fortuit. Il s'agit de «manifestantes ». La volonté 
de disqualification de l'État malien qui ne garantit pas la «liberté d'opinion » et qui réprime de manière "féroce" une manifestation féminine est évidente. Enfin, l'accusation de "terrorisme" dans un contexte ouest-africain, et même mondial, marqué par la prolifération des attaques revendiquées par les mouvements terroristes, n'est pas sans conséquence pour la cible dont l'image se trouve ternie. La référence à la problématique des droits de l'homme pointe vers de nombreux instruments juridiques nationaux et internationaux, au service ici de la défense et de la protection des femmes. En jouant sur ce tableau et en montrant que l'État malien déroge au respect de ces principes quasi-universels, le discours du MNLA lui dénie la qualité d'État moderne et le discrédite ainsi aux yeux de la communauté internationale. Au-delà de la cible qu'il faut disqualifier, ces attaques s'adressent donc aussi au « tiers » dont le soutien est sollicité.

En outre, le discours polémique comporte aussi une forte implication du locuteur, implication qui se manifeste dans notre corpus par la nature des actes locutifs réalisés. L'investissement du locuteur dans son propos est perceptible dans sa façon de nommer et des désigner les êtres et les évènements. Dans leurs déclarations, les porte-parole du mouvement rebelle ont recours à un lexique révélateur d'un jugement émis par le locuteur sur la cible du discours. Ainsi, lorsqu'ils parlent de " pogroms ", pour qualifier les actes des forces maliennes, ou de "police politique », pour désigner les services de la "Sécurité d'État ", ou encore lorsqu'ils ont recours à la comparaison "à la manière de la radio mille collines ", volontairement ou non, le locuteur livre son point de vue et un jugement personnel. Ce point de vue ou cette opinion s'appuie sur la mémoire collective et sociohistorique inscrite dans le lexique. Celui-ci rappelle les tueries de masses historiquement établies et orientées contre respectivement les communautés juives, les opposants politiques, enfin le génocide Tutsi dans lequel la « radio mille collines " au Rwanda a joué un rôle majeur. Ces procédés de désignation traduisent donc toute l'aversion du locuteur pour les forces armées maliennes et pour la « presse locale. » Il s'agit d'un acte de communication consistant à présenter les choses sous un certain angle afin de dévoiler au lecteur ou à l'auditoire l'image que le locuteur souhaite lui faire voir. Alice Krieg-Planque parle de cadrage ${ }^{17}$ pour désigner cette pratique. S'ajoutent à cela des formes linguistiques marquées du sceau de la présence du locuteur comme les épithètes («douloureuse présence malienne »), et les adverbes de manière (« faussement »).

Ainsi, la question des droits humains, les abus de pouvoir, le non-respect des principes démocratiques et celle des médias aux ordres sont des topiques mobilisées par les locuteurs du MNLA dans une dynamique polémique qui permet d'accuser la cible du discours. Directement ou non, le Mali est accusé de tous les maux, ce qui dénote une volonté affichée de discréditer l'autre en faisant de lui l'incarnation du mal de par l'engagement affectif dans l'énoncé produit. Cette modalité du dire est propre au discours de combat, où l'enjeu est de faire adhérer le destinataire à des valeurs en mettant en parallèle ceux qui les défendent et ceux qui les transgressent. On note ainsi une forte tendance à faire le procès du "gouvernement malien ». Cela donne lieu au recours de structures linguistiques ayant trait à l'accusation. Une accusation formulée à l'encontre d'une entité sur laquelle le locuteur n'hésite à porter un jugement dépréciatif. On est donc face à une interaction de registres mêlant à la fois l'accusation et le blâme. Ainsi, on note le foisonnement du lexique et des tournures réprobatives, évaluatives et délibératives. De nombreuses séquences sont marquées par la modalité déclarative portant sur les actions du Mali contre "l'Azawad», et les "populations 
azawadiennes, notamment touarègues et arabes ». L'ensemble de ces affirmations répétées à longueur de communiqués et de déclarations est destiné à véhiculer et à conforter l'opinion selon laquelle le "Mali est bel et bien un exemple de pays d'échec ». Par ailleurs, les désignations du gouvernement malien dans le discours du MNLA, les qualifications de ses agissements cherchent à présenter le Mali comme la source de tous les malheurs pour le «peuple de l'Azawad ». Ainsi, il ressort des mots et expressions mis en italiques dans les séquences citées ci-dessus, qu'il est l'occupant, le prédateur, le déstabilisateur, l'impotent, le barbare, le pyromane qui joue au pompier. Le lexique évaluatif qui se manifeste dans les adjectifs qualificatifs trahit tout l'investissement du locuteur dans son discours. Inversement, les rebelles sont présentés comme des acteurs conséquents qui prennent les décisions correspondant aux exigences de la situation, soucieux des intérêts du «peuple de l'Azawad » et qui invitent donc à soutenir leurs actions placées sous l'angle de l'impérieuse nécessité. En un mot, selon le locuteur, tout le mal provient du Mali.

Les faits linguistiques qui construisent ces différents éléments montrent que les locuteurs du MNLA ont recours aux appareils formels construisant les registres à la fois judiciaire, épidictique et délibératif. En d'autres termes, il y a une forte interaction des registres de la rhétorique aristotélicienne qui fait du discours une réalité à triple entrée : on prouve l'illégitimité du pouvoir du "gouvernement du Mali » pour traiter les questions de "l'Azawad ", on blâme le Mali et ses dirigeants et on loue les leaders du MNLA. À l'opposé d'un discours d'attaque, se construit un autre qui porte sur le mouvement lui-même ou sur l'Azawad inscrit dans une dynamique de mise en relief des valeurs défendues par le MNLA ou du "peuple de l'Azawad». Ce dernier est régulièrement désigné avec des termes élogieux à l'image de "Vaillant Peuple de l'Azawad », du " grand peuple de l'Azawad », du « vaillant peuple Azawadien ${ }^{18}$ ». Dans le même temps, le mouvement procède à une présentation de soi comme compatissant, sensible au malheur d'autrui. Dans la déclaration du 23 octobre 2013, Mossa Ag Attaher, Chargé de communication ${ }^{19}$, utilise les expressions «Le MNLA a appris avec tristesse ", " Le Mouvement National de Libération de l'Azawad témoigne en cette douloureuse occasion sa solidarité ", traduisant la compassion du locuteur. Celui-ci est affecté par la douleur vécue par l'autre, en l'occurrence le Tchad dont les troupes ont été victimes d'un attentat que le locuteur qualifie de "douloureuse». Le substantif "tristesse", l'épithète " douloureuse », permettent au locuteur de construire l'émotion qu'il ressent face à la situation décrite par l'expression «faisant des morts et blessés ». Cet extrait dévoile l'image d'un mouvement compatissant fortement affecté par les souffrances d'autrui. Par ailleurs, en déclarant :

Le MNLA tient, néanmoins, à attirer l'attention de l'opinion publique internationale que certains milieux malintentionnés cherchent à lui faire endosser régulièrement la responsabilité de certains actes auxquels il est complètement étranger dans le but manifeste de porter préjudice à son image de mouvement aspirant noblement aux valeurs de la paix et de la convivialité étant entendu que ces manœuvres n'ont d'autre but que de casser l'harmonie qui caractérise tant les populations de l'Azawad et de semer le doute dans les esprits ${ }^{20}$.

17 Le mouvement rebelle cherche à véhiculer une image de pacifiste. Dans un contexte de confrontation armée où il se trouve être celui qui a ouvert les hostilités, on comprend aisément que cette image (discursive) de pacifisme est destinée à contrer la possible image inverse d'un mouvement belliqueux. Le but visé serait alors de modifier le regard porté sur l'action du MNLA et sur le MNLA lui-même. Le constat ici est que pendant 
qu'il déploie toute sa verve pour disqualifier le gouvernement malien, en suscitant des sentiments d'hostilité à l'égard de celui-ci, le mouvement rebelle s'évertue à se présenter en alternative et en faiseur de paix. Le discours oscille ainsi entre susciter d'un côté indignation et répulsion, et de l'autre confiance et admiration, comme nous allons le voir dans les parties suivantes.

\section{Le registre élégiaque dans le discours du MNLA}

Nous employons "élégiaque» au sens défini plus haut par Marc Bonhomme: "se plaindre de x pour l'élégiaque, être ému par x pour le pathétique ». Plutôt qu'élégiaque, on pourrait dire aussi lamentation. L'essentiel est de voir qu'il ne s'agit pas ici à proprement parler de lamentation sur soi, mais sur son peuple.

munication du MNLA se déploie dans un contexte de guerre auquel se trouve nécessairement liée beaucoup de souffrance humaine. Le mouvement rebelle revendique l'indépendance d'un territoire qu'il considère en situation d'annexion par une force d'occupation. À ses yeux, ces situations riment toujours avec des abus opérés par l'occupant. Pour tenir compte ou afin d'exploiter ces données (vraies ou supposées), les communicants du mouvement rebelle produisent un discours qui met l'accent sur les souffrances habituelles de populations vivant sous occupation. De cette orientation découle un ensemble de traits linguistiques empreints d'une tonalité de complainte qui permet d'évoquer la présence du registre élégiaque construit autour des souffrances des populations ou encore « du peuple de l'Azawad».

De nombreux textes et déclarations font ainsi état du « calvaire du peuple Azawadien », de la « répression du peuple Azawadien et l'occupation de ses terres », du

comportement agressif à l'égard du peuple Azawadien à savoir : les assassinats, les arrestations et jugements arbitraires, l'interdiction des protestations pacifiques, l'établissement de listes des recherchés et l'excès de l'usage de force,

des "droits historiques confisqués en particulier la souveraineté entière sur sa terre ", "d'un peuple sous oppression, occupation et racisme", "confronté à toutes formes des pratiques rejetées internationalement "; ou encore la référence aux " 50 années de souffrance du peuple azawadien ${ }^{21} »$. On observe la récurrence du champ lexical de la souffrance infligée à des populations qui ne demandent qu'à vivre tranquillement sur leur terre. La souffrance est à la fois physique, morale et psychologique et le locuteur joue sur le contraste entre le droit élémentaire auquel aspire le "peuple Azawadien » et le niveau de cruauté auquel il est soumis depuis plus d'un demi-siècle. Il y ajoute les brimades dont il est victime afin d'émouvoir l'auditoire et d'obtenir ainsi toute sa compassion et son attention. On le voit, le jeu sur le pathos est très net. De la plainte sur son propre sort (registre élégiaque, se plaindre de $x$ ), on passe très naturellement à la tentative pour émouvoir le public international, le tiers (registre pathétique, être ému par $x$ ).

Par rapport à la bibliographie mentionnée sur le registre élégiaque, il faut souligner une différence notable. Ici, la complainte est collective puisque traitant de souffrances elles-mêmes collectives. On est évidemment en dehors de la thématique amoureuse qui, traditionnellement, domine dans l'élégie. Si l'on nous accorde cette inflexion, qui sort le registre élégiaque du seul contexte de la complainte amoureuse, on peut à partir d'une analyse lexicale, établir que le discours du MNLA relève en ce sens du registre élégiaque. En tout état de cause, la collectivité de la complainte est mise en évidence par le recours au pronom personnel «nous» dans les discours du MNLA. Cet ethos 
collectif élaboré est destiné à resserrer les liens communautaires entre populations touarègues victimes de l'action du Mali. La conviction du locuteur est également construite en discours à travers la modalité épistémique introduite par les expressions « il est certain », « il est aussi indéniable » et la répétition du verbe "savoir » conjugué au présent, « chacun de nous sait », " Ainsi nous savons ", et au passé composé, «nous l'avons $s u »$. Ces déclarations épistémiques sur les agissements du Mali sont renforcées par la négation non seulement de la volonté du Mali ("s'il veut donner») mais aussi de sa capacité à avoir quelque impact que ce soit sur l'Azawad (le Mali «ne peut rien faire »). Ces manifestations langagières du savoir sont adressées à la fois à l'auditoire composé du «peuple de l'Azawad» et de la communauté internationale. Selon les deux auditoires déjà indiqués, d'un côté le registre élégiaque permet de créer chez les populations de l'Azawad ou de renforcer leur sentiment d'appartenance à un groupe (et la nécessité de défendre une cause collective) ; et, de l'autre côté, le registre pathétique vise à rallier la communauté internationale, prise comme arbitre dans le conflit.

\section{L'appel à l'action ou le registre délibératif}

Dans cette logique de conquête de l'opinion internationale, il s'agit de lui montrer la justesse du combat mené par la rébellion. Cela conduit les communicants du MNLA à recourir au registre délibératif. Le cadre de production du discours implique donc trois acteurs, qui sont le locuteur, la cible et le tiers à qui le message est destiné. Le registre délibératif met au centre de l'activité discursive le tiers dont une action est attendue. La manifestation langagière de ce registre se voit dans la récurrence des formules langagières à forte teneur illocutoire :

Nous demandons [...]

Nous en appelons [...]

Nous, Mouvement national de libération de l'Azawad (MNLA), alertons [...] sur les dérives et les exactions répétées de l'armée malienne sur les civils depuis le 17 janvier dernier ${ }^{22}$.

Ces discours sont dominés par le recours aux verbes directifs (demandons, d'exiger, en appelons, alertons, condamne et dénonce), dont le but illocutoire est de mettre l'interlocuteur dans l'obligation de réaliser une action afin de transformer la réalité conformément à la volonté, au souhait, au désir du locuteur. Ces traits linguistiques, à la limite de l'exhortatif, relève du registre délibératif parce qu'ils mettent le destinataire dans une disposition d'action. Il lui est conseillé voire même commandé d'agir ou d'adopter une certaine attitude face à la situation initialement décrite. Certes, ce constat d'exhortation peut être fait aussi pour les adresses destinées « au peuple de l'Azawad » qui est invité à continuer la lutte, à resserrer ses rangs, et à faire preuve de courage. Mais il faut bien voir que cette même dynamique d'exhortation court dans les messages adressés aux défenseurs des droits de l'homme, à l'opinion internationale qui doit prendre la décision d'agir pour parer au plus pressé et protéger les populations dont la vie est en jeu. De manière générale, les discours diffusés décrivent une situation de péril pour les populations, posant du coup la nécessité d'agir par devoir moral pour les personnes interpellées. Il y a dans cette démarche un recours perceptible de mise en jeu de la modalitée ${ }^{3}$ déontique faisant appel à la notion d'obligation consécutive au rang, à la fonction, et aux valeurs défendues par le sujet interpellé. d'éviter que l'État malien ne se donne à un génocide» ou la demande d'intervention à 
l'endroit des défenseurs des droits de l'homme (« et demande aux organisations locales et internationales de droits de l'homme d'intervenir pour la libération de ces personnes innocentes, avant d'être soumises à une traditionnelle liquidation exercée par l'État du Mali et de ses services de sécurité à l'encontre du peuple de l'Azawad depuis plus de 50 ans »), ou encore la demande d'agir "face à l'utilisation des hélicoptères de guerre contre des populations civiles " lancée aux "Pays de la sous-région et la communauté internationale", l'appel "aux organisations locales et internationales de droits de l'homme » et enfin, l'« appel solennel et urgent à tous les défenseurs de la liberté à se montrer solidaire avec ces personnes arrêtées " placent les acteurs ainsi apostrophés dans l'obligation déontique d'agir non seulement pour les raison évoquées par le locuteur, mais aussi et surtout pour préserver l'image liée à la position sociale de l'individu, du sujet ou de l'institution concernée.

Registre polémique, complaintes et appel à l'action interagissent savamment dans le discours du MNLA. Cette mise en synergie répond à des visées discursives qu'il importe maintenant de rechercher.

\section{Registres discursifs au service des visées discursives des communicants du MNLA}

27 Les productions discursives du MNLA s'inscrivent dans un contexte de joute où l'enjeu est de justifier un acte (déclenchement de la guerre), de consolider un esprit communautaire au sein d'un groupe et autour d'une cause, de déconstruire l'image d'un adversaire désigné et, dans le même temps, se construire une réputation d'acteur légitime. Afin de rendre compte de de ces différentes fonctionnalité discursives, notre étude recourt maintenant à la notion de visée discursive qui prend en compte à la fois la proposition de visée argumentative formulée par R. Amossy et celle de visée de justification et de positionnement émise par Marc Angenot. Ces deux visée découlent, selon Angenot, du fait que les locuteurs «argumentent pour se situer par rapport aux raisons des autres ${ }^{24} »$ ou encore « argumentent pour se justifier, pour se procurer face au monde une justification [...] inséparable d'un avoir-raison ${ }^{25} »$. Cela implique un usage orienté des ressources linguistiques en vue de produire des effets sur l'interlocuteur. D'où l'idée de visées discursives des communicants du mouvement rebelle. Les discours du MNLA ont une visée argumentative. Ce qui, selon Amossy, veut dire que chaque production discursive est soutenue par une intention consciente reposant sur des stratégies discursives dont l'objectif ultime est de faire adhérer l'auditoire à une position donnée dans le conflit qui est en cours. Ainsi, au-delà de la cohésion qu'il entend renforcer au sein de la communauté de victime créé, le discours du MNLA vise à discréditer le pouvoir central de Bamako, à le mettre au banc des accusés afin de le rendre illégitime dans la gestion du Nord Mali. Les diverses accusations formulées à son encontre justifient cette volonté. À terme, il faut le mettre hors jeu. Pour cela, les registres discursifs présents constituent pour le locuteur des outils de construction ou de déconstruction des images discursives des parties au conflit. Le blâme du Mali et les louanges du MNLA construisent un ethos négatif pour l'un et un ethos positif pour l'autre. Le registre polémique va dans le même sens en suscitant ou exprimant des sentiments hostiles à l'égard du Mali. Dans le même temps, l'exposition des souffrances des «Azawadiens" relève d'une stratégie de victimisation qui vise à fédérer les opinions autour de la victime. Il s'agit alors d'un recours au pathos pour émouvoir l'auditoire et, principalement, la communauté internationale dont le MNLA attend un 
soutien dans son combat. C'est d'ailleurs ce qui explique le comportement allocutif des locuteurs qui traverse l'ensemble des discours produits et qui se manifeste à travers les appellatifs et la modalité interpellative. Il y a lieu de préciser que le recours à la victimisation contribue aussi à justifier l'entrée en guerre contre l'autorité gouvernementale.

L'ensemble des registres discursifs s'intègre dans un discours de justification de l'action militaire initiée contre le pouvoir central et les revendications sécessionnistes. La logique est donc de rendre acceptable l'usage des armes comme moyen d'expression politique en confirmant le bien fondé de l'action du MNLA, lequel passerait ainsi du statut de rebelle à celui de défenseur des droits d'une communauté victime d'acharnement. Mieux, il s'agit de mettre en avant le sens des responsabilités des acteurs du MNLA : ceux-ci interviennent parce qu'il y a un désordre consécutif, selon les discours produits, à l'incapacité de l'État malien d'assurer la sécurité, de lutter contre le terrorisme et de pacifier le Sahel. La décision de revendiquer l'autodétermination, selon ces séquences, est soutenue par le constat d'échec de l'autorité. Il y a donc un vide qu'il importe de combler afin d'assurer la sécurité et de «mettre un terme définitif aux atteintes portées sur les personnes et sur les biens des ressortissants étrangers ». Cette orientation discursive vise à établir toute la légitimité des revendications sécessionnistes. Dans le même temps, il s'agit de promettre un avenir radieux non seulement pour les populations locales, mais aussi et surtout pour les «ressortissants étrangers». On notera ici le choix d'élaborer une parole de promesse permettant aux communicants du MNLA de se poser en sujet politique défendant un idéal qu'ils proposent à l'auditoire. Le MNLA, par ce choix discursif, s'inscrit dans une perspective persuasive. N'étant pas dans une position d'autorité lui permettant d'en imposer aux acteurs régionaux et internationaux qui interviennent dans la crise malienne, le mouvement rebelle cherche à influencer par des moyens discursifs les décisions et les actions des intervenants, notamment celles de la communauté internationale. Le discours essaie alors de placer cette initiative sous l'angle de l'impérieuse nécessité où le mal et son incarnation, selon le discours du MNLA, doivent être mis hors de « l'Azawad » pour garantir le bien-être des populations civiles. Dans la même veine, les discours produits entendent positionner le MNLA dans l'espace politique. Ce positionnement prend en compte les questions liées aux droits de l'homme, à la protection des populations civiles, à la sécurité de la zone sahélienne et plus largement à la stabilité de la région ouest-africaine en proie à de nombreuses convulsions politiques mais aussi terroristes. Il faut donc faire admettre à tous que le MNLA est l'alternative ou une alternative crédible, et qu'il représente une solution politique, un acteur responsable avec des capacités d'organisation face à la situation.

Ce regard analytique porté sur les productions discursives du MNLA aura permis de mettre en avant, à partir des traits formels identifiés, les aspects d'une parole qui se situe au carrefour d'une diversité de registres dont notamment le polémique, le délibératif et l'élégiaque, entendu au sens de lamentation non sur soi mais sur le collectif. L'étude a établi aussi que cette interaction des registres n'implique nullement une dispersion. Bien au contraire, l'ensemble des registres mis en œuvre convergent vers une visée argumentative, une visée de positionnement et une visée de justification destinées non seulement à détruire un adversaire en construisant de lui un éthos négatif mais surtout à consacrer la légitimité et la crédibilité du mouvement rebelle. 
Cette parole au carrefour de divers registres permet au locuteur de mettre en œuvre plusieurs modalités. Cela lui permet à la fois de justifier son action, de se montrer crédible et légitime, d'adresser une parole de promesse susceptible de fédérer les opinions autour de son initiative et de déconstruire l'image de l'autre c'est-à-dire le pouvoir central de Bamako.

\section{BIBLIOGRAPHIE}

Amossy, R., 2008. " Modalités argumentatives et registres discursifs : le cas du polémique ", Les registres. Enjeux stylistiques et visées pragmatiques, Lucie Gaudin-Bordes et Geneviève Salman dir., Louvain-la-Neuve, Academia Bruylant, p. 93-108.

Angenot, M., 2008. Dialogue de sourds. Traité de rhétorique antilogique, Paris, Mille et Une Nuits.

Boilley, P., 1999. Les Touaregs Kel Adagh : dépendances et révoltes : du Soudan français au Mali contemporain, Paris, Karthala.

Bonhomme, M., 2015. «L'interaction entre figuralité et registres discursifs », Pratiques [En ligne], 165-166 | 2015, mis en ligne le 01 octobre 2015, consulté le 27 mai 2016. URL : http:// pratiques.revues.org/2391

Galy, M., 2013. La guerre au Mali, Paris, la Découverte.

Gaudin-Bordes, L.et Salman, G. dir., 2008. Les registres. Enjeux stylistiques et visées pragmatiques, Louvain-la-Neuve, Academia Bruylant.

GEMDEV et Université du Mali, 2005. Mali France. Regards sur une histoire partagée, Bamako / Paris, Donniya / Karthala.

Krieg-Planque, A., 2013. Analyser les discours institutionnels, Paris, A. Colin.

Langage et société, 2008/2, $\mathrm{n}^{\circ}$ 124, « Les registres de discours : une notion en débat ».

Maingueneau, D., 2008. «Les trois dimensions du polémique », Les registres. Enjeux stylistiques et visées pragmatiques, Lucie Gaudin-Bordes et Geneviève Salman dir., Louvain-la-Neuve, Academia Bruylant, p. 109-120.

Meunier, A., 1974. « Modalités et communication ». Langue française, $n^{\circ} 21$, p. 8-25.

Philippe, G., 2008. « Registres, appareil formels et patrons », dans Les Registres : enjeux stylistiques \& visées pragmatiques, Lucile Gaudin et Geneviève Salvan dir., Louvain-la-Neuve, Academia Bruylant, p. 27-37.

\section{NOTES}

1. G. Philippe, "Registres, appareils formels et patrons ", dans L. Gaudin et G. Salvan dir., Les Registres : enjeux stylistiques \& visées pragmatiques, Louvain-la-Neuve, Academia Bruylant, 2008, p. 27-37.

2. Ibid., p. 31 . 
3. Langage et société, 2008/2, n 124, «Les registres de discours : une notion en débat ».

4. D. Maingueneau, «Les trois dimensions du polémique », dans L. Gaudin et G. Salvan dir., Les Registres : enjeux stylistiques \& visées pragmatiques, op. cit., p. 110.

5. M. Bonhomme, "L'interaction entre figuralité et registres discursifs ", Pratiques [En ligne], 165-166 | 2015, mis en ligne le 01 octobre 2015, consulté le 27 mai 2016. URL : http:// pratiques.revues.org/2391.

6. Se référer pour cela au communiqué $\mathrm{n}^{\circ} 1$ du MNLA publié le 16 octobre 2011 , communiqué que l'on pourrait qualifier d'acte de naissance du MNLA.

7. Capitale du Mali, État de l'Afrique de l'Ouest.

8. Communiqué publié le 16 octobre 2011.

9. GEMDEV et Université du Mali, Mali-France. Regards sur une histoire partagée, Bamako/Paris, Donniya/Karthala, 2005.

10. P. Boilley, Les Touaregs Kel Adagh: dépendances et révoltes: du Soudan français au Mali contemporain, Paris, Karthala, 1999.

11. M. Galy, La guerre au Mali, Paris, la Découverte, 2013.

12. Cité par R. Amossy dans son article « Modalités argumentatives et registres discursifs : le cas du polémique ", dans L. Gaudin et G. Salvan dir., Les Registres : enjeux stylistiques \& visées pragmatiques, op. cit., p. 93-108.

13. Marc Bonhomme, op. cit.

14. Communiqué mis en ligne sous le titre : SEMAINE $N^{\circ 05-27 / 12 / 2012, ~ L u n d i, ~} 27$ Février 2012, 00:48, Pour le MNLA, Khoumeidy Ag Acharatmane. URL : http://www.mnlamov.net/actualites/ 34-actualites/136-semaine-nd05-25122012.html.

15. Communiqué du MNLA : Journée Internationale de la Femme Jeudi, 08 Mars 2012, 02:02.

16. R. Amossy, «Modalités argumentatives et registres discursifs : le cas du polémique », op. cit., p. 105.

17. A. Krieg-Planque, Analyser les discours institutionnels, Paris, Armand Colin, 2013, p. 94.

18. Déclaration faite à Ouagadougou, le 25 septembre 2013, par Bilal Ag ACHERIF, Secrétaire Général du Mouvement National De Libération de l'Azawad, MNLA.

19. Déclaration du MNLA faite à Kidal le 23 octobre 2013, par Mossa Ag Attaher, Chargé de communication.

20. Déclaration du MNLA faite à Ouagadougou le 20 octobre 2013, par Mossa Ag Attaher, Chargé de communication.

21. Déclaration du MNLA faite à Ouagadougou le 29 septembre 2013, par Mossa Ag Attaher, Chargé de communication.

22. Communiqué du MNLA $\mathrm{n}^{\circ} 04$ en date du 22/01/2012, 13:22. URL: https:// azawadinfo.wordpress.com/2012/01/22/communique-n-04-012012-sur-les-exactions-commisespar-larmee-malienne-sur-les-populations-de-lazawad/.

23. A. Meunier, « Modalités et communication », Langue française, 1974, $\mathrm{n}^{\circ}$ 21, p. 8-25.

24. M. Angenot, Dialogue de sourds. Traité de rhétorique antilogique, Paris, Mille et Une Nuits, 2008, p. 441 .

25. Ibid., p. 441. 


\section{AUTEUR}

NANOUROUGO COULIBALY

Université Félix Houphouet-Boigny de Cocody 